Britain. (Some of the results of these measurements have since been published; see Nature, 170, 916; 1952.)

The study of solid reactions is probably one of the main applications for radioactive tracers in physical chemistry ; and in Session 26, on chemical applications, the first paper, by Dr. R. Lindner, dealt with this subject. Another interesting paper in this Session, by members of the Kodak Research Laboratories, was on the determination of silver in photographic images using radioactive iodide (iodine131). Where a large number of analyses is required, the radio-iodination method has a distinct advantage over the potentiometric method and is to be preferred where only a small image is available, as in X-ray crystallographic patterns, or when the optical density is low.

The remaining four Sessions (27-30) were devoted respectively to ion exchange, non-destructive testing, static elimination and radioactivation analysis. Mr. K. Fearnside, of Isotope Developments, Ltd., Aldermaston, described four instruments : the transmission beta-ray thickness gauge used in paper, plastic sheet and metal foil production; the reflexion beta-ray gauge for the measurement of the thickness of coatings and liquid films; the gamma-ray thickness gauge applied to thick or hot-rolled metal strip ; and the package monitor used to check the contents of sealed packages as they leave the packaging machine. Mr. G. Syke dealt in more general terms with the problem of inspection and gauging with ionizing radiations. Fog-making on certain kinds of fabrics due to static electricity forming on the fibres can be prevented by dissipating the static when it occurs before it has time to become a nuisance. The static may be made to leak away either through the textile itself or through the surrounding air. Ionizing the air by means of a radioactive substance is a most successful method; and in his paper on radioactive static eliminators for the textile industry, Dr. P. S. H. Henry, of the Shirley Institute, discussed thoroughly the question of static on textiles and the relative merits of alpha- and beta-ray static eliminators. Finally, Mr. A. A. Smales gave some indications of the scope of radioactivation analysis, the essential basis of which is that an element is detected and determined by the formation of a radioactive nuclide which is then subjected to radioassay, and Prof. G. Chaudron described some work on the analysis of traces of sodium and copper in high-purity aluminium by pile activation.

\section{BREEDING-BACK OF THE TARPAN}

$\mathrm{B}$ FORE the existence of human culture a number of different kinds of wild horses existed in Europe and Asia. Of these only one is extant to-day. This one was discovered by the Russian Przewalski in the desert-like steppes of Zungaria in western Siberia. Like most steppe-dwelling animals, the Przewalski wild horse is red-brown in colouring and light underneath. As a legacy from its forbears it still carries zebra-like stripes at the backs of the legs which show up very clearly in the summer coat. The discovery of the last remaining species of wild horse was followed by the news that in the 1920's it had become extinct in the free state. To-day only a few are left scattered in various zoological gardens, and none is breeding except in the sixteen-head Munich herd.
It is on this herd that the hopes of preserving the species must rest.

If the species is not preserved, apart from its own interest, one of the most interesting animals of to-day will have become extinct. One of the European wild horses, the mouse-grey, for which the Russian name of tarpan has been adopted, was, in historical times, still extant over large areas of Europe (Oryx (Journal of the Fauna Preservation Society), 1, No. 7 ; November 1952).

In the first half of the past century it was still numerous in the steppes of the Ukraine north of the Black Sea. In 1876 it became extinct, leaving few traces of its existence. The tarpan was different from the Przewalski wild horse not only in colouring but also in build. The Przewalski has strong thick bones and an almost disproportionately heavy skull with narrow forehead which, many investigators believe, is the reason why the heavy carthorses of to-day are descended from this type. The tarpan has much finer bones and a short light head and is probably the progenitor of swift riding horses.

Distinguished for his successful efforts in breeding back the wild aurochs, Heinz Heck, director of the Tierpark Hellabrunn, Munich, has also successfully bred-back the tarpan. Mares from Iceland and Gothland that were markedly like tarpans were crossed with a Przewalski stallion; the resultant crosses were mated with one another and, in 1933, from this stock emerged the first foal with a mousegrey tarpan colouring. Other foals followed, and through resultant crossings there is to-day a considerable stock of back-bred tarpons in the Tierpark, Hellabrunn.

\section{MICROSCOPY OF PLANT ASHES}

7 TE statement that a microscope magnifies four hundred times never quite conveys a true idea of the wealth of detail that can be seen at this magnification. Perhaps only when the incredibly small fragments of material on a cosh, the minute amounts of blood or hair and other such negligible 'small dust of the balance', appear as important items of evidence in criminal proceedings is it realized how valuable is this instrument in the examination of such unpromising material.

One would, however, be pardoned in assuming that even the microscopist would have to admit defeat when dealing with material that had been so thoroughly incinerated that only an ash remained. However, in 1920, the Austrian botanist Hans Molisch investigated the structures left after incinerating plant material and showed that the ash still retained definite structural features. Prof. Gosta Edman, now professor of botany and pharmacognosy in the Royal Pharmaceutical Institute, Stockholm, was then a pupil of Molisch and later set out to investigate these 'ash pictures' systematically and has since published numerous papers on the subject. Recently he was awarded the Hanbury Memorial Medal of the Pharmaceutical Society of Great Britain which, in conjunction with the Linnean and Chemical Societies, periodically makes the award for "high excellence in the prosecution or promotion of original research in the Natural History and Chemistry of Drugs". After receiving the medal, Prof. Edman gave an account of his work on ash pictures (Pharm. J., 170,189 ; 1953). 